\title{
COPING RELIGIOSO/ESPIRITUAL E CÂNCER DE MAMA: UMA REVISÃO SISTEMÁTICA DA LITERATURA
}

\author{
Carina Maria Veit ${ }^{1}, \&$ Elisa Kern de Castro ${ }^{2}$ \\ 1- RS, Brasil; 2- UNISINOS, São Leopoldo, Brasil
}

\begin{abstract}
RESUMO- Esta revisão sistemática da literatura teve por objetivo examinar pesquisas publicadas entre 2006-2011 que investigaram o coping religioso/espiritual (CRE) em mulheres com câncer de mama e seus efeitos na adaptação à doença. Foram utilizados os descritores religious beliefs, spirituality, religiosity, religious experiences, religion, coping behavior, adjustment, breast cancer, religious coping e spiritual coping nas bases de dados LILACS, Medline, PshycInfo e Web of Science. Destaca-se a primazia do padrão positivo de CRE quando comparado ao negativo. Três estudos não demonstraram relação entre o CRE positivo e o bem-estar físico e psicológico, ao passo que o CRE negativo esteve associado a um pior ajustamento psicológico em cinco estudos.

Palavras-chave- Coping religioso/espiritual; Câncer de mama; Revisão sistemática da literatura
\end{abstract}

\section{SPIRITUAL/RELIGIOUS COPING AND BREAST CANCER: A SYSTEMATIC LITERATURE REVIEW}

\begin{abstract}
This systematic literature review aimed to examine the research published between 2006 and 2011 that investigated religious/spiritual coping (RSC) in female breast cancer patients and its effects in adaptation to the disease. The descriptive categories used were 'religious beliefs', 'spirituality', 'religiosity', 'religious experiences', 'religion', 'coping behavior', 'adjustment', 'breast cancer', 'religious coping' and 'spiritual coping' based on data from LILACS, Medline, PsychInfo and Web of Science. We highlight the superiority of the positive RSC over negative RSC. Three studies did not show any relation between positive RSC and physical and psychological well-being, whereas negative RSC was associated with worse psychological adjustment in five studies.
\end{abstract}

Keywords- Religious/spiritual coping; Breast cancer; Systematic literature review.

Recebido em 26 de Novembro de 2012/ Aceite em 05 de Dezembro de 2012

O câncer é um conjunto de mais de 200 doenças que têm como característica comum o crescimento anormal de células que invadem diferentes partes do corpo e que podem proliferar-se (metástase), destruindo os tecidos circundantes e afetando o funcionamento do organismo (Speechley \& Rosenfield, 2000). O câncer de mama é uma das mais comuns neoplasias humanas e sua etiologia é multifatorial, envolvendo alimentação, fatores reprodutivos e desequilíbrios hormonais (Fabri, Carcangiu, \& Carbone, 2008). As células que revestem os ductos mamários e que formam o câncer de mama são normalmente ordenadas em conteúdo e disposição, sendo reconhecidas através de sua característica de calcificação (Fallowfield \& Clark, 2002). 
O Coping $^{l}$ pode ser definido como o conjunto de estratégias cognitivas e comportamentais que são utilizadas pelas pessoas em resposta a alguma situação estressante, ou seja, são as tentativas elaboradas pelos indivíduos para preservar a sua saúde mental e física em circunstâncias adversas (Antoniazzi et al., 1998; Lazarus \& Folkman, 1984; Straub, 2005). Para selecionar as respostas que serão utilizadas para lidar com um evento estressor, o indivíduo realiza duas avaliações, a primária e a secundária. $\mathrm{Na}$ avaliação primária, o indivíduo analisa se a circunstância é potencialmente prejudicial e ameaçadora, revelando quão importante é este evento para o seu bem-estar. Se a situação é compreendida como algo nocivo, o indivíduo inicia a avaliação secundária, na qual examina os recursos disponíveis para lidar com o evento estressante (Snyder \& Dinoff, 1999). Por vezes, o controle da situação está além das condições dos indivíduos e o coping se apresenta mais como uma forma de lidar com a situação do que propriamente de resolvê-la (Curtis, 2000). Há, portanto, uma importante distinção entre as estratégias de coping que são direcionadas ao manejo e resolução da situação estressante e aquelas em que o objetivo é a regulação da resposta emocional do problema. Essas formas são referidas, respectivamente, como coping focalizado no problema e coping focalizado na emoção (Lazarus \& Folkman, 1984).

A literatura relacionada ao coping concentra-se na área da Psicologia Clínica da Saúde, de forma especial nos casos de doenças crônicas e intervenções médicas (Faria \& Seidl, 2005; Straub, 2005), e articula conhecimentos provindos de áreas como Psicologia, Fisiologia, Psiconeuroimunologia e Antropologia (Paiva, 2007). A saúde e o bem-estar são os resultados mais adequados do coping, contudo, nas situações em que a forma de lidar com um evento da vida é mal adaptativa, essa pode causar efeitos adversos à saúde física e emocional (Shaw, 1998).

A religião e a espiritualidade são, por muitas vezes, formas adotadas para lidar com o estresse gerado pelo câncer de mama (Astrow, Wexler, Texeira, He, \& Sulmasy, 2007). Contudo, cabe destacar que esses conceitos, ainda que se sobreponham, apresentam características distintas. Conforme Miller e Thoresen (2003), existem problemas quando esses constructos são igualados ou separados, sendo frequentemente abordados em conjunto na maioria dos contextos. Diante disso, os autores definem religião como um fenômeno institucional, social e delimitado, que inclui práticas, crenças e modos de organização específicos, e que é centralmente preocupado com a espiritualidade. A espiritualidade, por sua vez, seria o interesse pela vida e pelo imaterial, que se reflete no modo de viver, no emocional e social. Miller e Thoresen (2003) exemplificam a complexidade da diferenciação e da sobreposição desses conceitos quando afirmam que o campo da religião é a espiritualidade, assim como o campo da medicina é a saúde. Pargament (1997) definiu coping religioso ${ }^{2}$ como o uso da fé, religião ou espiritualidade no manejo das situações estressantes ou dos momentos de crise que ocorrem ao longo da vida. Segundo Koenig (2008), frequentemente, as pessoas

\footnotetext{
${ }^{1}$ A palavra coping pode significar "enfrentar", "lidar com” ou "adaptar-se a" (Antoniazzi, Dell'Aglio \& Bandeira, 1998). Contudo, essa é considerada um vazio linguístico, já que não é possível encontrar, no português, uma palavra que a traduza em sua complexidade. Devido a isso, o uso do termo "enfrentamento", correspondente a sua tradução no português, será evitado neste artigo.

${ }^{2}$ Neste artigo, os autores não realizam uma distinção entre religião e espiritualidade. O termo espiritualidade é utilizado para referir a uma das funções principais da religião: o esforço para estabelecer um relacionamento com o sagrado. Dessa forma, a espiritualidade estaria imbuída na religião, não apresentando um caráter distinto. Conforme Panzini (2004), esta diferenciação iniciou apenas em 1997 e, por isto, publicações mais antigas referem o coping apenas como religioso.
} 


\section{COPING RELIGIOSO/ESPIRITUAL E CÂNCER DE MAMA}

dependem de suas crenças e práticas religiosas para lidar com as adversidades da vida, sendo essas promotoras de um senso de controle e de auxílio que encoraja a tomada de decisões e facilita o processo de coping.

Embora, durante muito tempo, ciência e religião tenham sido consideradas áreas contraditórias, foi possível, neste milênio, visualizar uma abertura à investigação dos aspectos religiosos envolvidos na vida humana (Sousa, Tillman, Horta, \& Oliveira, 2004). Há um aumento de pesquisas que têm investigado o papel da religião e da espiritualidade no contexto da saúde (Faria \& Seidl, 2005; Paiva et al., 2009), as quais têm indicado que o envolvimento religioso, geralmente, está associado com a saúde física e mental, afetando os resultados em saúde (Koenig, 2007). Em revisão de literatura (brasileira e internacional) abrangendo os temas qualidade de vida e espiritualidade, os artigos encontrados nas bases de dados PsycINFO e PubMed/Medline, entre 1979 e 2005, evidenciaram a existência de uma forte associação entre esses temas, o que, por sua vez, tem resultado em discussões sobre a inclusão da dimensão espiritual no conceito de saúde, indo além do bem-estar físico, mental e social (Panzini, Rocha, Bandeira, \& Fleck, 2007). Entretanto, os autores apontam que, no Brasil, as pesquisas voltadas ao impacto da religiosidade na qualidade de vida e nas relações com a saúde ainda são escassas, sugerindo que novos estudos sejam desenvolvidos, a fim de que esses forneçam dados empíricos que auxiliem no planejamento de práticas em saúde embasadas espiritualmente.

Os fatores religiosos e espirituais têm sido associados a diversos aspectos de adaptação ao diagnóstico e tratamento do câncer, indicando sua importância para a saúde e para a recuperação dos pacientes (Al-Azri, Al-Awisi, \& Al-Moudhri, 2009; Alcorn et al., 2010; Barros, 2008; Feher \& Maly, 1999; Lin, \& Bauer-Wu, 2003; Mytko \& Knight, 1999; Schnoll, Harlow, \& Brower, 2000; Soothill, Morris, Harman, Thomas, Francis, \& McIllmurray, 2000; Stefanek, McDonald, \& Hess, 2005; Thuné-Boyle, Stygall, Keshtgar, \& Newman, 2006; Yanez et al., 2009). De fato, a maioria das pesquisas tem destacado em seus resultados a prevalência do uso positivo do coping religioso/espiritual (CRE) quando comparado à sua utilização de uma forma negativa (Gall, Guirguis-Younger, Charbonneau, \& Florack, 2009; Hebert, Zdaniuk, Schulz, \& Scheier, 2009; Thuné-Boyle, Stygall, Keshtgar, Davidson, \& Newman, 2011; Zwingmann, Wirtz, Müller, Körber, \& Murken, 2006; Zwingmann, Müller, Körber, \& Murken, 2008).

A revisão sistemática de literatura realizada por Thuné-Boyle et al. (2006) investigou o uso de estratégias de CRE e seus efeitos na adaptação à doença, bem-estar psicológico e qualidade de vida em pacientes com câncer. A busca em quatro bases de dados, compreendida entre os anos de 1977 a 2004, resultou na análise de 17 artigos. Desses, dez investigaram especificamente alguns tipos de câncer, sendo que a maioria era de mama. A análise dos artigos permitiu verificar que, embora muitos estudos americanos não tenham encontrado resultados significativos, um número equivalente de pesquisas encontraram evidências de que o CRE é benéfico no processo de adaptação ao câncer. Apenas três estudos encontraram efeitos prejudiciais no uso dessa estratégia de coping, sendo que um deles tinha por objetivo investigar o CRE negativo. No que se refere aos estudos europeus, nenhum concluiu que o CRE era importante. Tais resultados, como sugerido por Thuné-Boyle et al. (2006), podem ter ocorrido devido às deficiências metodológicas, principalmente no que diz respeito à mensuração e conceitualização do CRE. 
A revisão sistemática de Thuné-Boyle et al. (2006) ilustra a escassez de estudos que tratam da temática do CRE em pacientes com câncer, já que, dos 17 artigos selecionados, 10 não examinaram o CRE como objetivo principal. Com vistas a sistematizar os dados já existentes, e focando em um determinado tipo de câncer, esta revisão sistemática da literatura tem por objetivo examinar os efeitos do CRE na saúde psicológica de mulheres com câncer de mama que vivenciam o período do diagnóstico e/ou do tratamento ou, ainda, que sejam sobreviventes à doença. A partir das categorias analisadas na revisão sistemática de ThunéBoyle et al. (2006), pretende-se investigar: a) as associações existentes entre o CRE (positivo ou negativo) e a adaptação à doença; b) como os estudos têm medido o CRE; e c) as principais limitações metodológicas dos estudos envolvidos nessa revisão.

\section{MÉTODO}

Critérios de Inclusão/Exclusão dos Artigos

a) os artigos devem examinar o papel da religiosidade/espiritualidade como uma estratégia de coping em mulheres que tenham sido recentemente diagnosticadas com câncer de mama, que estejam em fase de tratamento ou que sejam sobreviventes à doença; b) os artigos devem ser quantitativos e devem medir o uso da religiosidade/espiritualidade no processo de enfrentamento do câncer de mama (variável independente), sendo o bem-estar psicológico e/ou adaptação à doença utilizados como variáveis dependentes; c) os artigos devem ser provenientes de revistas científicas datadas dos últimos seis anos (2006 - 2011) e os termos coping religioso (religious coping) ou coping religioso/espiritual (religious/spiritual coping) devem aparecer no título, no resumo ou nas palavras-chave dos artigos; d) foram excluídos os artigos que não apresentavam acesso disponível ao texto completo pelo portal da CAPES e que não foram enviados pelos autores após solicitação via e-mail, e produções oriundas de congressos (resumos, resumos expandidos ou textos completos), dissertações de mestrado, teses de doutorado, livros ou capítulos de livros, resenhas, comentários de artigos e editoriais; e) foram excluídos os artigos resultantes de estudos com metodologia qualitativa, artigos de validação/adaptação de instrumentos e revisões teóricas e/ou sistemáticas da literatura; f) foram excluídos os artigos que não estavam em Inglês, Espanhol, ou Português.

\section{Bases de Dados}

As pesquisas foram feitas nas bases de dados LILACS, Medline, PshycInfo e Web of Science e os descritores utilizados foram: religious beliefs, spirituality, religiosity, religious experiences, religion, coping behavior, adjustment, breast cancer, religious coping e spiritual coping. Em cada base de dados foram feitas as seguintes combinações dos descritores: "religious beliefs and breast cancer", "spirituality and coping behavior and breast cancer", "religiosity and coping behavior and breast cancer", "religious experiences and coping behavior and breast cancer", "adjustment and spiritual coping or religious coping and breast cancer", "religious coping and breast cancer" e "spiritual coping and breast cancer".

\section{Procedimentos de Organização do Material}




\section{COPING RELIGIOSO/ESPIRITUAL E CÂNCER DE MAMA}

A Figura 1 ilustra os procedimentos realizados para a seleção do material. A pesquisa feita na base de dados LILACS não obteve resultados.

Figura 1. Critérios de Seleção dos Artigos para a Revisão Sistemática

\section{$1^{\circ}$ Passo: Realização das buscas nas bases de dados com as sete combinações de descritores}

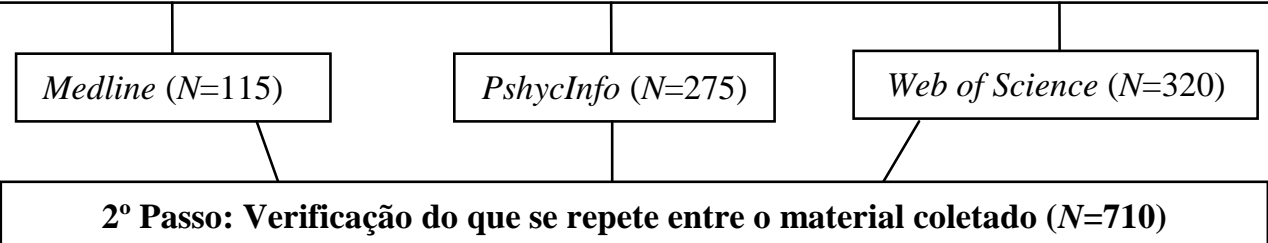

Passo: Verificação do que se repete entre o material coletado $(N=710)$

Material para ser selecionado $(N=290)$

$3^{\circ}$ Passo: Exclusão do material a partir dos critérios estabelecidos

I) Não apresentavam mulheres com câncer de mama na amostra ou no estudo $(n=186)$.

II) Artigos teóricos e/ou de revisão de literatura $(n=5)$ e qualitativos $(n=26)$.

III) Artigos de desenvolvimento, adaptação e/ou validação de instrumento $(n=3)$

IV) Dissertações de mestrado $(n=14)$, resumos de eventos $(n=2)$, resumos indisponíveis $(n=2)$ e comentário de artigo $(n=1)$.

V) Artigos sem os termos "coping religioso" (religious coping) ou "coping religioso/espiritual" (spiritual/religious coping) no título, no resumo ou nas palavras-chave $(n=39)$.

VI) Artigos que apresentaram o termo "religious coping” no resumo, mas que não tinham por objetivo investigar o papel da religião/espiritualidade como uma estratégia de coping $(n=2)$.

VII) Artigo que deveria ser incluído, mas que estava indisponível, mesmo após solicitação ao autor $(n=1)$.

VIII) Artigos para análise nessa revisão sistemática da literatura $(N=9)$ 


\section{Aspectos Gerais}

\section{RESULTADOS}

O quadro 1 apresenta a descrição das nove pesquisas encontradas, todas desenvolvidas em países da Europa (Aukst-Margetić, Jakovljević, Ivanec, Margetić, Ljubičić, \& Šamija, 2009; Laarhoven, Schilderman, Vissers, Verhagen, \& Prins, 2010, Thuné-Boyle et al., 2011; Zwingmann et al., 2006; Zwingmann et al., 2008) e da América do Norte (Gall et al. 2009; Hebert et al., 2009; Morgan, Gaston-Johansson, \& Mock, 2006; Schreiber, 2011), todos disponíveis na língua inglesa.

Tabela 1

Resumo dos Artigos Analisados

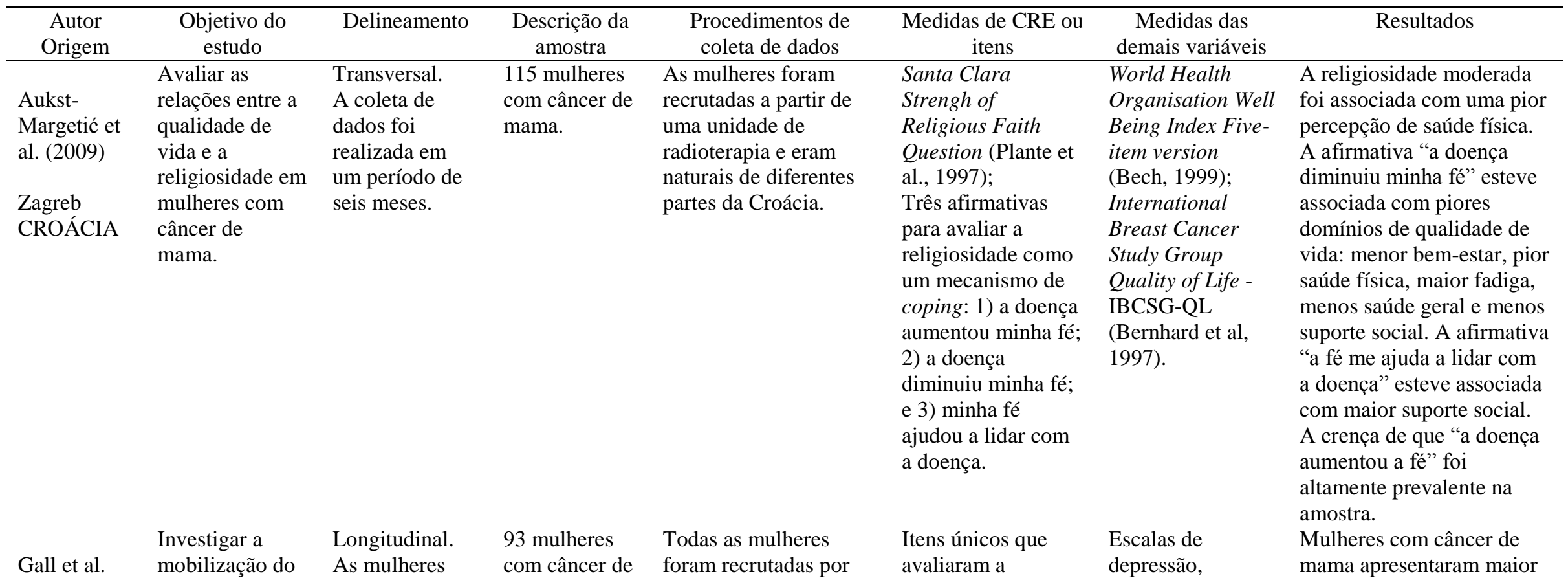


COPING RELIGIOSO/ESPIRITUAL E CÂNCER DE MAMA

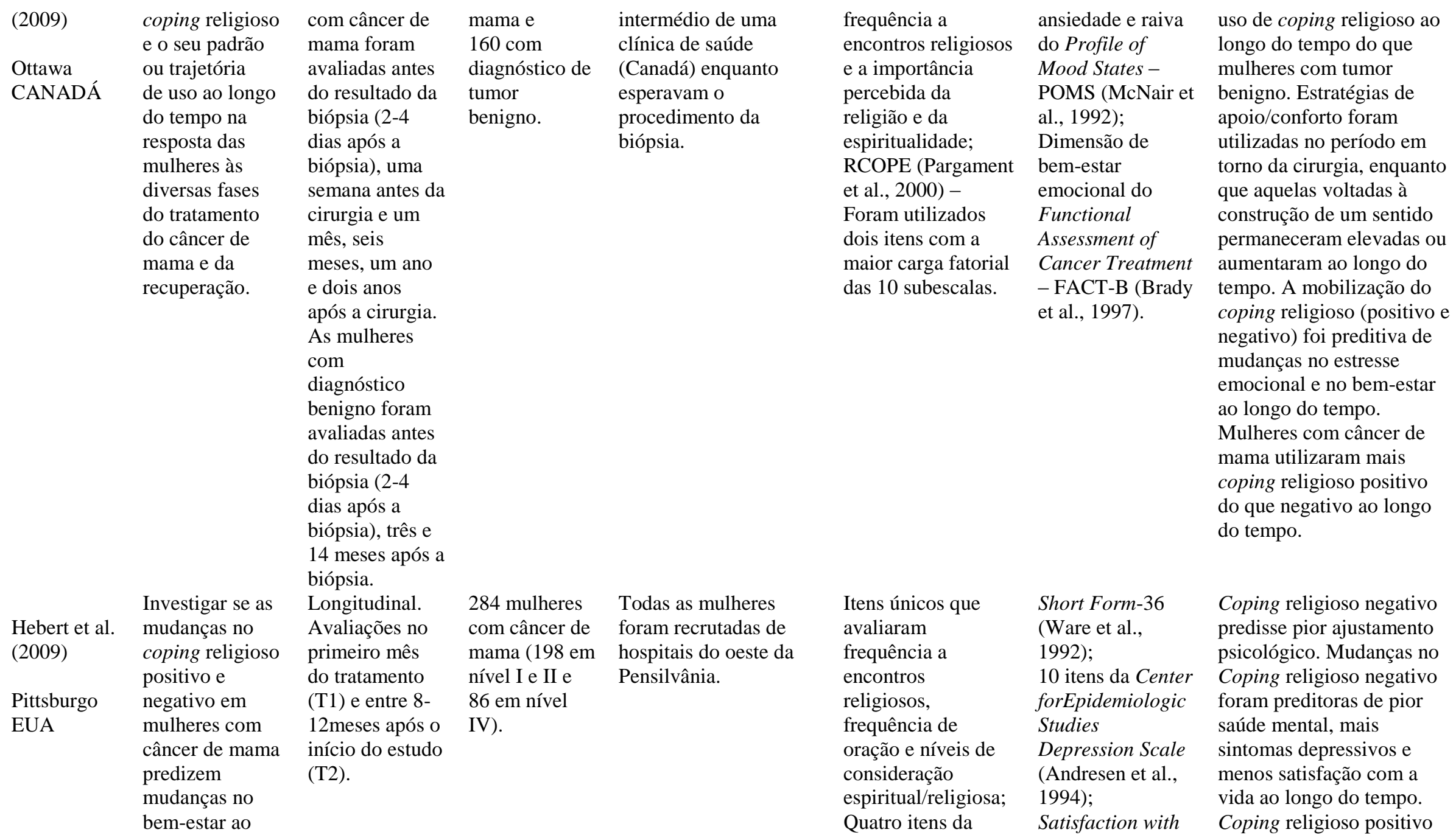


longo do tempo.

Examinar a
relação entre a

Laarhoven et al. (2010)

Nimegue

PAÍSES

BAIXOS

$\begin{array}{ll} & \text { ser conhecido e } \\ & \text { estratégias de } \\ & \text { coping em um } \\ & \text { grupo de } \\ & \text { pacientes } \\ & \text { paliativos de } \\ \text { câncer } & \text { holandeses que } \\ & \text { não estavam } \\ & \text { mais recebendo } \\ & \text { tratamentos } \\ & \text { antitumorais e } \\ & \text { que estavam } \\ \text { diante da morte. } & \text { Explorar as } \\ & \text { relações entre } \\ & \text { bem-estar } \\ & \text { espiritual, } \\ \text { Morgan et } & \text { coping religioso } \\ \text { al. (2006) } & \text { e qualidade de } \\ \text { Louisville } & \text { vida em } \\ \text { EUA } & \text { mulheres afro- }\end{array}$

$\begin{array}{ll}\text { Transversal. } & 68 \text { pacientes } \\ \text { A coleta de } & \text { sob cuidados } \\ \text { dados foi } & \text { paliativos } \\ \text { realizada de } & \text { (13\% de } \\ \text { julho de 2003 a } & \text { câncer de } \\ \text { dezembro de } & \text { mama). } \\ 2005 . & \\ \end{array}$

Os participantes foram recrutados de

departamentos de

medicina interna e

oncologia médica, de

uma unidade de

cuidados paliativos de uma universidade, de

dois hospitais gerais,

de dois hospitais que

cuidam de pacientes

terminais e de um

consultas de cuidados

paliativos nos Países

Baixos.

\section{Transversal,}

descritivo.

\section{As mulheres foram} recrutadas de centros
11 mulheres

diagnosticadas

pela primeira

vez com

câncer de

mama

recebendo

quimioterapia serviço regional de

RCOPE (Pargament et al., 2000), dois

de Coping

Religioso Positivo e

dois de Negativo.

Images of God

Scale (Van der

Vem, 1998).

\section{COPE-Easy \\ (Carver et al., \\ 1989).}

et 1985$)$

não apresentou associação significativa com o bemestar.

Uma imagem impessoal de Deus foi o preditor mais

relevante para o coping e esteve associada a quatro estratégias diferentes,

enquanto que uma imagem pessoal de Deus foi associada a uma estratégia, e a imagem que não pôde ser conhecida não apresentou nenhuma associação. A idade foi o preditor sociodemográfico mais importante para o coping e apresentou valor preditivo negativo para a busca de conselho e informação e por apoio moral. de oncologia e de consultórios de oncologistas dentro da costa Atlântica e Sudeste dos Estados Unidos. Os

BriefRCOPE
(Pargament et al.
1998).

The Functional
Assessment and
Cancer
TherapyScale -
FACT-B (Cella et
al., 1993),
incluindo nove
questões

As mulheres utilizaram mais coping religioso positivo do que negativo durante a fase de tratamento. Correlações positivas (moderadas a fortes) foram encontradas entre bem-estar espiritual e 
COPING RELIGIOSO/ESPIRITUAL E CÂNCER DE MAMA

americanas que

se encontravam

em tratamento

quimioterápico

radioterápico.

\section{Examinar o}

Transversal,

Schreiber, J. efeito das visões

A. (2011) de Deus em

sobreviventes de

Louisville câncer de

EUA mama, nas

estratégias de

coping

religioso,

depressão,

ansiedade,

estresse,

preocupações

sobre a

recorrência da

doença e bem-

estar

psicológico. ou

enviados por correio

àquelas que disseram

aos seus médicos que

concordariam em

participar do estudo.

Aquelas que

retornaram receberam

um cartão de telefone

no valor de 20 dólares.

As mulheres foram

\section{9}

obreviventes

de câncer de

mama que

a universidade

completaram o e de um consultório de

tratamento e

que estavam

em transição

do estágio de

tratamento

para o de

sobrevivência

inicial (6-30

meses após o

diagnóstico).

oncologia da

comunidade. Os

questionários foram

enviados por correio

aquelas que

participaram receberam

$\$ 10$. adicionais que se

referem

especialmente a

pacientes com

câncer de mama;

Domínio de

Qualidade de vida

da Spiritual Well

Being - SWB

(Brady et al.

1999).

Image of God Scale Versão Breve da

(Bader et al., 2006), Depression

Religious/Spiritual

Coping Short Form

(Pargament et al.

2000)
Anxiety Stress

Scale (Lovibond

et al., 1995),

Subescala de

medo global da

Concerns About

Recurrence Scale

(Vickberg, 2003)

Scales of

Psychological

Well-Being (Ryff,

1989). os domínios de qualidade

de vida física, emocional

bem-estar funcional. Não

foram encontradas

correlações significativas entre variáveis clínicas e os domínios da qualidade de

vida. Correlação negativa

(moderada) foi encontrada entre coping religioso

negativo e bem-estar físico.

Nenhuma relação

significativa foi encontrada

entre o bem-estar

psicológico e a subescala

de conservação espiritual

(RCOPE). Mulheres que

viam Deus como engajado

obtiveram maiores índices

de bem-estar e menores

escores de preocupações

sobre a recorrência da

doença, depressão,

ansiedade e estresse. As

mulheres que viam Deus

como engajado mantiveram

o bem-estar psicológico

até mesmo com o uso de

estratégias de conflito

espiritual. O principal fator associado ao bem-estar

psicológico foi a visão das

mulheres de um Deus

engajado, mais do que o 


$\begin{array}{ll}\text { Thuné- } & \begin{array}{l}\text { Examinar a } \\ \text { prevalência e o } \\ \text { Boyle et al. } \\ \text { curso de } \\ \text { estratégias de } \\ \text { coping religioso } \\ \text { em pacientes }\end{array} \\ \text { Londres } & \begin{array}{l}\text { recentemente } \\ \text { diagnosticadas } \\ \text { REINO }\end{array} \\ \text { UNIDO côncer de } \\ \text { mama no Reino } \\ \text { Unido. }\end{array}$

Longitudinal.

Avaliações

feitas três dias

após a cirurgia

(na maioria), e

três e 12 meses

após a cirurgia.

Unido.
155

participantes

recentemente

diagnosticadas

com câncer de

mama e com

cirurgia

subseqüente

(92\% estavam

no estágio I e

II).
A maioria (85\%) dos questionários foi

preenchida no quarto

do hospital e algumas

pacientes preferiram

responder em casa.

Não é descrito onde

foram feitas as

avaliações dos três e 12

meses após a cirurgia.
Três itens (com

maior carga

fatorial) de 13

subescalas da

RCOPE (Pargament

et al., 2000).
Investigar o Transversal.

papel do coping A coleta de

et al. (2006) religioso

dados foi
156 mulheres

\section{recentemente}

diagnosticadas
Todas as mulheres

foram recrutadas de um

centro de reabilitação
16 itens de coping

religioso $(8$

positivos e 8
Brief COPE

(Carver, 1997).

- tipo de estratégia de coping empregado.

O uso de estratégias de coping não religioso foi,

em geral, mais comum. E o coping religioso, apesar de ter sido usado por $66 \%$ da

amostra, foi a estratégia de coping menos utilizada quando a avaliação foi feita com uma medida geral

Brief COPE. As estratégias "coping religioso positivo e ativo" e "busca por apoio em líderes e membros do grupo religioso" foram significativamente maiores no período da cirurgia. Já as estratégias "sentir-se

punido e abandonado por

Deus" e "busca por

purificação espiritual"

foram significativamente

maiores em torno da

cirurgia e 12 meses

após.As estratégias de

coping religioso da Brief

COPE também

apresentaram índices

significativamente maiores

no período da cirurgia.

Duas subescalas

(Depressive

As participantes

apresentaram níveis

elevados de coping 
COPING RELIGIOSO/ESPIRITUAL E CÂNCER DE MAMA

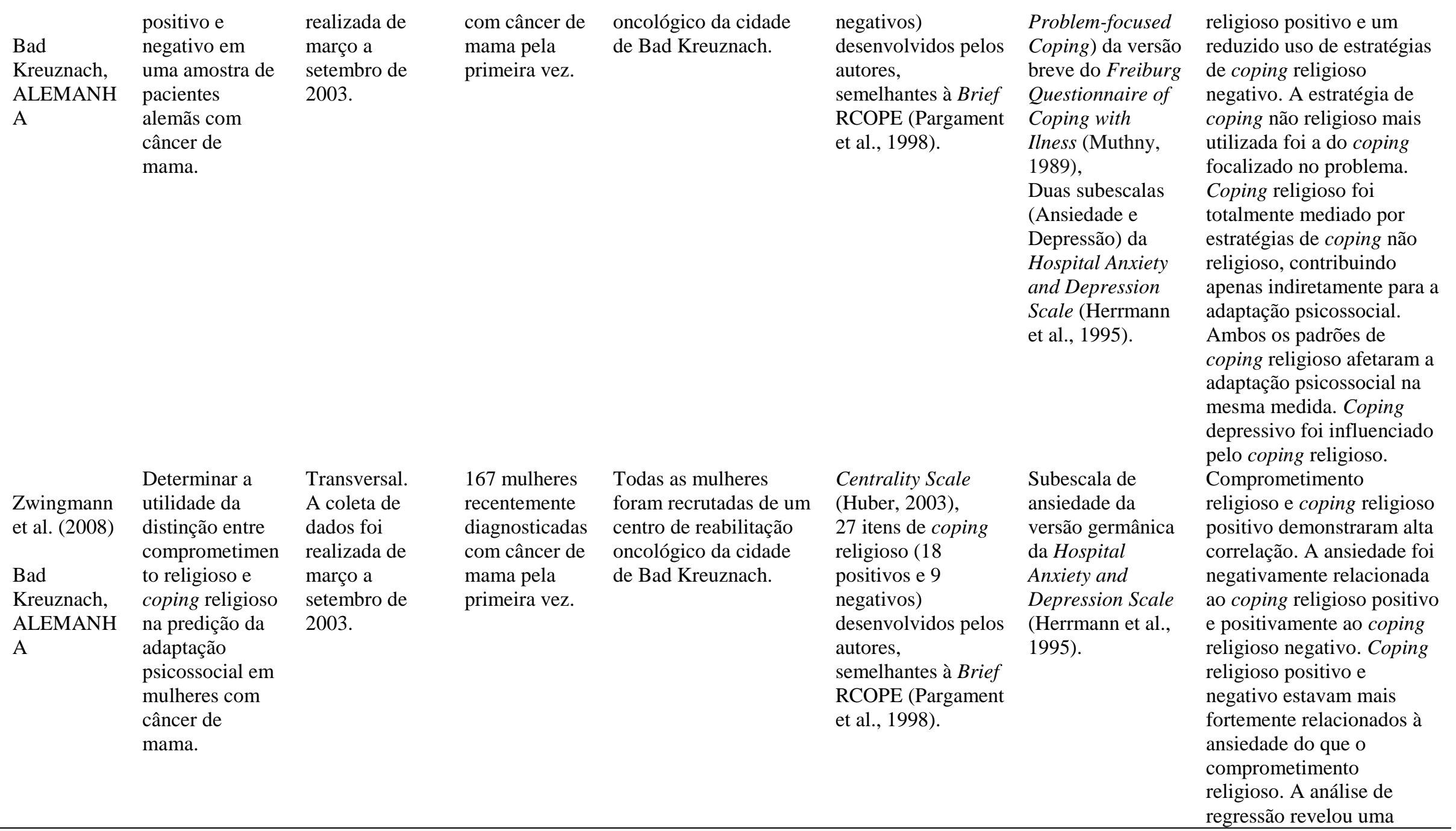




\section{Carina Veit e Elisa Castro}

interação entre

comprometimento religioso

e coping religioso negativo,

sugerindo uma relação

entre maiores níveis de

comprometimento religioso

e maiores níveis de coping

religioso negativo. 
Um dos estudos, desenvolvido no Reino Unido (Thuné-Boyle et al., 2011), destacou diferenças nos resultados quando comparados às pesquisas realizadas nos Estados Unidos. Ao contrário dos EUA, em que existe uma alta proporção de pessoas com uma determinada afiliação religiosa e que frequentam regularmente serviços religiosos, a população do Reino Unido apresentou, em geral, baixos níveis de envolvimento religioso/espiritual. Contudo, algumas mulheres que se declararam não religiosas relataram ter feito uma breve oração antes de irem para a sala de operação. Embora as estratégias de coping religioso negativo tenham sido menos comuns do que outras estratégias de coping não-religioso, essas corresponderam a $53,5 \%$ (Reavaliação dos poderes de Deus), indicando que mais da metade da amostra tinha algum conflito com a sua fé em relação ao diagnóstico do câncer de mama. Os resultados demonstraram que houve uma significativa redução nas estratégias de coping religioso ao longo do tempo, sugerindo que as pacientes estão mais propensas a buscar a ajuda de Deus nos primeiros estágios do curso da doença do que nos seguintes. As estratégias de coping religioso positivo foram significativamente maiores no período em torno da cirurgia do que aos três e 12 meses pós-cirúrgicos.

As estratégias de coping religioso também foram mobilizadas no período de adaptação à doença no estudo de Gall et al. (2009), sendo as estratégias relacionadas à busca de apoio e conforto elevadas no período em torno da cirurgia e reduzidas ao longo do tempo, enquanto que as estratégias de construção de sentido permaneceram elevadas ou aumentaram a longo prazo. Estratégias de coping religioso positivas e negativas foram preditoras de angústia. $\mathrm{O}$ aumento do descontentamento espiritual, por exemplo, foi preditor de um aumento na angústia emocional, enquanto que a entrega ativa e a ajuda religiosa predisseram uma diminuição no estresse. Da mesma forma, aspectos negativos e positivos do coping religioso estiveram associados ao bem-estar emocional: uma diminuição no descontentamento espiritual acompanhada do aumento da ajuda religiosa foi preditora de um aumento no bemestar emocional.

Em contrapartida, no estudo de Hebert et al. (2009), o coping religioso positivo não apresentou nenhuma associação com o bem-estar, ao passo que o coping religioso negativo foi preditor de pior saúde mental, maior depressão e menor satisfação de vida, indicando um pior ajustamento psicológico relacionado a esse tipo de estratégia. Conforme os autores, tais achados destacam a importância de interrogar as pacientes sobre possíveis conflitos religiosos/espirituais e, a partir disso, desenvolver intervenções que promovam o bem-estar emocional. Resultados semelhantes foram encontrados no estudo piloto de Morgan et al. (2006), realizado com mulheres afro-americanas. Os autores não descreveram nenhuma associação significativa entre as variáveis pesquisadas e o coping religioso positivo. Todavia, verificaram a existência de associações negativas entre o coping religioso negativo e o bemestar físico. Ademais, associações positivas entre bem-estar espiritual e os domínios de qualidade de vida (físico, emocional e bem-estar funcional) foram encontradas.

Somado a isso, Schreiber (2011) aponta para a busca de conhecimento das visões de mundo das pacientes, no intuito de facilitar o acesso aos recursos religiosos/espirituais que elas possam ter. A autora, ao investigar as implicações das visões de Deus na vida de sobreviventes de câncer de mama, encontrou diferenças significativas entre aquelas que viam Deus como alguém engajado (engaged), mas não naquelas que viam Deus como zangado (angry). Altos níveis de bem-estar psicológico e baixos índices de medo da recorrência da 


\section{Carina Veit e Elisa Castro}

doença, de depressão, ansiedade e estresse foram encontrados naquelas que viam Deus como altamente engajado, o que aponta para os efeitos potenciais da fé na adaptação psicológica. Laarhoven et al. (2010) também investigaram as imagens de Deus em pacientes sob cuidados paliativos (13\% com câncer de mama) e concluíram que a adesão a uma imagem impessoal de Deus foi preditora positiva para as estratégias de coping de "Busca por conselho e informação", "Busca de apoio moral" e "Negação", e preditora negativa para a estratégia "Humor". Em contrapartida, a adesão a uma imagem pessoal de Deus esteve associada a apenas uma estratégia, a única do instrumento relacionada a aspectos religiosos/espirituais: "Voltar-se à religião". Os autores apontam para a urgência de consideração dessas diferentes percepções de Deus nas pesquisas, tendo em vista as diferenças das crenças e práticas religiosas entre os países.

Em um estudo feito com pacientes alemãs, Zwingmann et al. (2006) descobriram que tanto o coping religioso positivo quanto o negativo contribuíram para o coping depressivo, mas não para o coping focalizado no problema. $\mathrm{O}$ coping depressivo foi predito negativamente pelo coping religioso positivo e positivamente pelo coping religioso negativo, o que indica que ambos os padrões de coping religioso predisseram a adaptação psicossocial. Embora o coping depressivo tenha sido menos utilizado nesse estudo, esse revelou-se como sendo um forte preditor para a ansiedade e depressão, sendo que o coping religioso correspondeu a $14 \%$ da variância do coping depressivo. O coping religioso negativo foi predito pela idade e pelo estado civil, demonstrando que mulheres mais velhas e sem parceiro apresentam mais coping religioso negativo. Já o coping religioso positivo não foi predito por nenhuma variável. Além disso, nenhuma relação significativa foi encontrada entre variáveis clínicas e os estilos de coping religioso.

Também realizado com mulheres alemãs, Zwingmann et al. (2008) verificaram que a ansiedade esteve negativamente relacionada ao coping religioso positivo e positivamente associada ao coping religioso negativo. O comprometimento religioso não apresentou associação significativa com a ansiedade, em contrapartida, apresentou uma forte associação com o coping religioso positivo. O coping religioso negativo, por sua vez, não apresentou associação com o comprometimento religioso, tampouco com o coping religioso positivo. Como no estudo de Zwingmann et al. (2006), não foram encontradas associações significativas entre variáveis clínicas e o coping religioso. Todavia, mulheres mais velhas apresentaram maior comprometimento religioso, maiores índices de coping religioso negativo e menor ansiedade, enquanto as mulheres com maior nível de escolaridade ou que moravam com um companheiro obtiveram um menor índice do coping religioso negativo. De modo particular, esse estudo apresentou diferenças relacionadas à afiliação religiosa e demais variáveis investigadas, demonstrando que mulheres católicas $(45 \%)$ relataram maiores níveis de comprometimento religioso, maiores índices de coping religioso positivo e menor ansiedade, enquanto que as protestantes (38\%) demonstraram maior coping religioso negativo. De acordo com os autores, o uso do padrão negativo de coping religioso foi relativamente baixo na amostra.

Por fim, Aukst-Margetić et al. (2009) constataram que mulheres com maior religiosidade estavam convencidas de que a fé ajudou-as na cura da doença e que a concordância com essa afirmativa estava positivamente associada com o apoio social que um envolvimento religioso oferece. Além disso, a percepção de que a fé ajudou a lidar com o câncer esteve fortemente 
apoiada entre aquelas mulheres que tinham mais filhos. Já a concordância com a afirmativa de que a doença diminuiu a fé esteve fortemente associada com piores índices de qualidade de vida. Neste estudo, destacou-se o alto índice da afirmativa "a doença aumentou a minha fé", enfatizando a importância da religiosidade no processo de coping e adaptação ao câncer de mama.

\section{Variáveis Clínicas}

Somente uma das pesquisas (Laarhoven et al., 2010) não foi realizada por uma amostra composta exclusivamente por mulheres com câncer de mama. Três foram realizadas com mulheres recentemente diagnosticadas (Thuné-Boyle et al., 2011; Zwingmann et al., 2006; Zwingmann et al., 2008), duas com pacientes em qualquer estágio da doença (Aukst-Margetić et al., 2009; Hebert et al., 2009), uma com mulheres antes e depois do procedimento da biópsia (Gall et al., 2009), uma com pacientes sob cuidados paliativos (Laarhoven et al., 2010), uma com mulheres diagnosticadas com a doença pela primeira vez e que estavam recebendo tratamento quimioterápico ou radioterápico (Morgan et al., 2006) e uma com sobreviventes do câncer de mama (Schreiber, 2011).

Dentre os estudos que apresentaram o nível do câncer das participantes, todos tinham como maioria os estágios iniciais da doença: $33 \%$ em estágio I e 48,7\% em estágio II (AukstMargetić et al., 2009), 75,3\% entre os estágios 0 e II (Gall et al., 2009), 70\% em estágio I e II (Hebert et al., 2009), 72,1 \% em estágio II (Morgan et al., 2006), 92\% em estágio I e II (Thuné-Boyle et al., 2011), 31\% em estágio I e 47\% em estágio II (Zwingmann et al., 2006) e $30 \%$ em estágio I e 47\% em estágio II (Zwingmann et al., 2008). Schreiber (2011) e Laarhoven et al. (2010) não apresentaram nenhuma variável clínica.

Hebert et al. (2009) não encontraram particularidades no coping religioso entre as participantes em diferentes estágios do câncer. Em contrapartida, Gall et al. (2009) verificaram diferenças nos níveis de coping religioso entre mulheres nos estágios avançados e iniciais da doença. Todavia, optaram por combinar os grupos nas análises, uma vez que os padrões de mudança no coping religioso haviam sido muito similares ao longo do tempo entre as participantes, não existindo associações significativas entre o estágio do câncer e a angústia emocional e o bem-estar. Corroborando com esses resultados, encontram-se Zwingmann et al. (2006) e Zwingmann et al. (2008), que não encontraram nenhuma associação significativa entre as variáveis clínicas (estágio da doença, tempo de diagnóstico e procedimento cirúrgico) e as religiosas, e Morgan et al. (2006), que não encontraram associação significativa entre variáveis clinicas (estágio da doença e tempo de diagnóstico) e o bem-estar espiritual.

Apenas uma pesquisa (Morgan et al., 2006) detalhou as combinações de tratamentos vivenciadas pelas pacientes $(63,6 \%$ cirurgia e quimioterapia e $36,4 \%$ cirurgia, quimioterapia e radioterapia), uma variável clínica que deveria ser incluída pelos estudos quando o tema de investigação é justamente o processo de coping/enfrentamento da doença. Cinco estudos apresentaram algumas informações: Aukst-Margetić et al. (2009) recrutaram para o estudo mulheres da unidade de radioterapia, as quais diferiam do tratamento recebido anteriormente; Gall et al. (2009) descreveram que 57\% das participantes tinham realizado mastectomia e que $50,6 \%$ tinham variadas combinações de tratamento após a cirurgia, tais como quimioterapia, radioterapia e hormonoterapia; Thuné-Boyle et al. (2011) citaram que $61 \%$ das participantes haviam sido submetidas à mastectomia conservadora e que as demais haviam sofrido algum outro tipo de mastectomia, existindo um total de $40 \%$ da amostra que realizara esvaziamento 
das axilas em nível II; Zwingmann et al. (2006) e Zwingmann et al. (2008) relataram que praticamente todas as participantes do estudo haviam realizado cirurgia de mastectomia.

Variáveis Relacionadas à Religião

Praticamente todas as pesquisas apresentaram as percentagens quanto à filiação religiosa das participantes, sendo, dentre elas, predominantes a Igreja Católica (Gall et al., 2009; Laarhoven et al., 2010; Zwingmann et al., 2006; Zwingmann et al., 2008), a Protestante (Hebert et al., 2009; Schreiber, 2011; Thuné-Boyle et al., 2011) e a Batista (Morgan et al., 2006). Apenas o estudo de Aukst-Margetić et al. (2009) não apresentou esse dado, justificado pelo fato da população da Croácia ser predominantemente Católica (87,98\%).

Somado a isso, Gall et al. (2009) avaliaram a frequência a encontros religiosos e a importância percebida da religião/espiritualidade na vida das participantes; Hebert et al. (2009) verificaram, além da frequência a encontros religiosos, a frequência de oração e os níveis de consideração espiritual/religiosa; Zwingmann et al. (2008) mediram o comprometimento religioso (grau de religiosidade que guia a vida diária de alguém).

Medidas de Avaliação de Coping Religioso/Espiritual

Três estudos (Gall et al, 2009; Hebert et al., 2009; Thuné-Boyle et al., 2011) foram realizados com itens selecionados da RCOPE (Pargament, Koenig \& Perez, 2000). Já Zwingmann et al. (2006) e Zwingmann et al. (2008) optaram por desenvolver itens semelhantes aos encontrados na Brief RCOPE (Pargament et al., 1998), que poderiam se encaixar ao contexto religioso/cultural de seu país, uma vez que ainda não estão disponíveis na Alemanha instrumentos que avaliem o coping religioso no seu respectivo idioma.

Morgan et al. (2006) e Schreiber (2011) foram os únicos pesquisadores que utilizaram uma escala completa para a avaliação do coping religioso, a BriefRCOPE (Pargament et al., 1998) e a Religious/Spiritual Coping Short Form - RCOPE - (Pargament et al., 2000), respectivamente. Além disso, Schreiber (2011) procurou identificar como as sobreviventes de câncer de mama viam a Deus e o que elas creem que Ele faz no mundo, através do Image of God Scale - IGS (Bader, Dogherty, Froese, Johnson, Mencken, Park \& Stark, 2006), um instrumento desenvolvido em Waco (Texas) que permite a identificação de quatro visões de Deus: benevolente, autoritário, crítico e distante. De modo similar, Laarhoven et al. (2010) utilizaram a Images of God Scale (Van der Vem, 1998), uma escala holandesa que avalia a adesão a uma imagem de Deus pessoal, impessoal e/ou que não pode ser conhecido e que tem sido utilizada, dentre outros, em um grande estudo $(n=1008)$ sobre o desenvolvimento sociocultural nos Países Baixos.

Aukst-Margetić et al. (2009), de forma particular, utilizaram o Santa Clara Strengh of Religious Faith Question - SCSORF - (Plante \& Boccaccini, 1997), um instrumento que mede a força das crenças religiosas independentemente da denominação ou afiliação religiosa e que já foi validado em pacientes com câncer. Nesse estudo, o uso da religiosidade como um mecanismo de coping foi avaliado a partir de três afirmativas, respondidas a partir de uma escala Likert de quatro pontos: "a doença aumentou minha fé", "a doença diminuiu minha fé" e "minha fé ajudou a lidar com a doença".

\section{Características Metodológicas}

Dentre os estudos analisados, três eram longitudinais (Gall et al, 2009; Hebert et al., 2009; Thuné-Boyle et al., 2011) e os demais transversais (Aukst-Margetić et al., 2009; Laarhoven et al., 2010; Morgan et al., 2006; Schreiber, 2011; Zwingmann et al., 2006; Zwingmann et al., 
2008). Esse dado vai ao encontro do observado na revisão sistemática de literatura de ThunéBoyle et al. (2006), em que a maioria $(n=9)$ das pesquisas eram transversais.

No que se refere ao tamanho das amostras, verificou-se que o número de participantes dos estudos variou entre 11 e $284(M=148,6, D P=84,1)$. Dois estudos longitudinais apresentaram as taxas de perda ao longo do tempo: Gall et al. (2009) tiveram perdas de 24,4\% e de $23,4 \%$ em sua amostra ao longo de dois anos, sendo essas de mulheres com câncer de mama e com diagnóstico benigno, respectivamente. Os autores verificaram que as participantes do estudo não diferiram das desistentes em termos de idade, importância religiosa e espiritual, angústia e bem-estar no pré-diagnóstico (2-4 dias após a biópsia). Contudo, no que tangia ao coping religioso, as participantes demonstraram um menor uso da delegação passiva (coping religioso negativo) no pré-diagnóstico do que as desistentes; Thuné-Boyle et al. (2011) tiveram uma perda de $19 \%$ em sua amostra ao longo de um ano e observaram que a única diferença entre as mulheres que continuaram no estudo em relação às mulheres desistentes era o nível de escolaridade, sendo as médias em anos de estudo de 14,9 e 12,5, respectivamente.

Além disso, foi possível observar que todas as informações foram coletadas em clínicas e/ou hospitais, com exceção de Morgan et al. (2006) e Schreiber (2011), que enviaram o material por correio, oferecendo incentivo financeiro para a participação.

\section{DISCUSSÃO}

As pesquisas que tiveram por objetivo avaliar o coping religioso encontraram a primazia do padrão positivo quando comparado ao negativo (Gall et al, 2009; Hebert et al., 2009; Morgan et al., 2006; Schreiber, 2011; Thuné-Boyle et al., 2011; Zwingmann et al., 2006; Zwingmann et al., 2008). No entanto, em alguns estudos o coping religioso positivo não apresentou nenhuma relação com o bem-estar físico e psicológico (Hebert et al., 2009; Morgan et al., 2006; Schreiber, 2011), ao passo que o coping religioso negativo esteve associado ao aumento de angústia emocional (Gall et al., 2009), de coping depressivo (Zwingmann et al., 2006) e de ansiedade (Zwingmann et al., 2008) e de pior ajustamento psicológico (Hebert et al., 2009) e bem-estar físico (Morgan et al., 2006).

Somado a isso, foi possível observar que nesses estudos (Gall et al, 2009; Hebert et al., 2009; Morgan et al., 2006; Schreiber, 2011; Thuné-Boyle et al., 2011; Zwingmann et al., 2006; Zwingmann et al., 2008) os instrumentos escolhidos para avaliar o coping religioso foram apropriados, já que foram utilizados itens selecionados da RCOPE Scale (Pargament et al., 2000) ou a escala completa. Conforme Thuné-Boyle et al. (2006), a RCOPE Scale (Pargament et al., 2000) oferece uma medida baseada teoricamente que examina toda a gama de estratégias benéficas e prejudiciais de coping religioso, abordando tal constructo de uma forma multidimensional. Em contrapartida, no estudo de Aukst-Margetić et al. (2009), o coping religioso foi medido através de três afirmativas, que parecem não abranger a complexidade de tal conceito. Como o estudo de Laarhoven et al. (2010) não tinha por objetivo avaliar o coping religioso de um modo específico, a escala utilizada foi a de COPEEasy (Carver, Scheier,\& Weintraub, 1989), que inclui aspectos religiosos.

No que se refere às principais limitações metodológicas dos estudos analisados, destacamse: o delineamento transversal (Aukst-Margetić et al., 2009; Laarhoven et al., 2010; Morgan 


\section{Carina Veit e Elisa Castro}

et al., 2006; Schreiber, 2011; Zwingmann et al., 2006; Zwingmann et al., 2008), a ausência de controle de comorbidades e de variáveis psicológicas em mulheres em diferentes momentos de tratamento da doença (Aukst-Margetiæ et al., 2009; Hebert et al., 2009), a impossibilidade de saber por que as pacientes que receberam o material por correio não responderam (Schreiber, 2011), a amostra ser composta apenas por mulheres recém diagnosticadas e, por conseguinte, com melhor prognóstico (Thuné-Boyle et al., 2011; Zwingmann et al., 2006; Zwingmann et al., 2008), a mensuração da adaptação psicossocial através de subescalas de ansiedade e depressão (Zwingmann et al., 2006; Zwingmann et al., 2008), a perda de participantes que na avaliação inicial haviam apresentado maiores índices de delegação passiva (Gall et al., 2009), e, por fim, a impossibilidade de generalização dos dados devido ao número reduzido das amostras e às diferenças culturais e religiosas.

Sugere-se que futuros estudos tenham por objetivo específico a avaliação do coping religioso/espiritual em mulheres com câncer de mama. Tendo em vista o quão invasivos são os tratamentos de câncer, bem como os seus respectivos impactos físicos e psicológicos nas áreas da sexualidade, maternidade e feminilidade (Silva, 2008; Muller, Hoffman,\& Fleck, 2006; Wanderley, 2003), sugere-se que esses venham a explorar de uma forma mais detalhada as implicações das variáveis clínicas no processo de coping religioso/espiritual, tais como tempo de diagnóstico, tipo de tratamento realizado, gravidade da doença, recorrência do câncer, etc. Além disso, sugere-se que sejam feitos estudos com pacientes em fase terminal, a fim de investigar a interação dos fatores religiosos/espirituais quando do risco eminente de morte.

O presente estudo revisou, exclusivamente, os artigos que apresentavam os termos "coping religioso" ou "coping religioso/espiritual" no título, no resumo ou nas palavraschave, como feito por Thuné-Boyle et al. (2006). Devido a isso, é provável que artigos que investiguem o coping religioso através de escalas abrangentes de coping tenham ficado excluídos da análise. O material aqui encontrado confirma a escassez de estudos nessa área, tanto que optou-se por incluir na análise o estudo de Laarhoven et al. (2010), que apresentava uma amostra com diferentes tipos de câncer. Não obstante, destaca-se que o termo "religious/spiritualcoping" foi escrito apenas no resumo de Thuné-Boyle et al. (2011), razão pela qual, ao discutir os resultados deste trabalho, foi utilizado o termo "coping religioso".

\section{REFERÊNCIAS}

Al-Azri, M., Al-Awisi, H., \& Al-Moudhri, M. (2009). Coping with a diagnosis of breast cancer-literature: Review and implications for developing countries. The Breast Journal, 15, 615-622. doi: 10.1111/j.1524-4741.2009.00812.x

Alcorn, S. R., Balboni, M. J., Prigerson, H. G., Reynolds, A., Phelps, A. C., Wright, A. A.,... Balboni, T.A. (2010). "If God wanted me yesterday, I wouldn't be here today': Religious and spiritual themes in patients' experiences of advanced cancer. Journal of Palliative Medicine, 13, 581-588. doi: 10.1089/jpm.2009.0343

Andresen, E. M., Malmgren, J. A., Carter, W. B., \& Patrick, D. L. (1994). Screening for depression in well older adults: Evaluation of a short form of the CES-D (Center for Epidemiologic Studies Depression Scale). American Journal of Preventive Medicine, 10, 77-84. 
Antoniazzi, A. S., Dell'Aglio, D. D., \& Bandeira, D. R. (1998). O conceito de coping: Uma revisão teórica. Estudos de Psicologia, 3, 273-294. doi: 10.1590/S1413294X1998000200006

Astrow, A. B., Wexler, A., Texeira, K., He, M. K., \& Sulmasy, D. P. (2007). Is failure to meet spiritual needs associated with cancer patients' perceptions of quality of care and their satisfaction with care? Journal of Clinical Oncology, 25, 5753-5757. doi:10.1200/JCO.2007.12.4362

*Aukst-Margetić, B., Jakovljević, M., Ivanec, D., Margetić, B., Ljubičić, Đ., \& Šamija, M. (2009). Religiosity and quality of life in breast cancer pacients. Collegium Antropologicum, 33, 1265-1271.

Bader, C., Dogherty, K., Froese, P., Johnson, B., Mencken, F.C., Park, J. Z., \& Stark, R. (2006). American piety in the 21st century: New insights to the depth and complexity of religion in the U.S. Texas: Baylor Institute for Studies of Religion.

Barros, A. C. S. D. (2008). Câncer de mama. In V. A. Carvalho, M. H. P. Franco, M. J. Kovács, R. P. Liberato, R. de C. Macieira, M. T. Veit, et al. (Eds.), Temas em psicooncologia (pp.40-45). São Paulo: Summus.

Bech, P. (1999). Health-related quality of life measurements in the assessment of pain clinic results. Acta Anaesthesiologica Scandinavica, 43, 893-896. doi: 10.1034/j.13996576.1999.430906.x

Bernhard, J., Hiirny, C., Coates, A. S., Peterson, H. F., Castiglione-Gertsch, M., Gelber, R., ... \& Rudenstam, C. M. (1997). Quality of life assessment in patients receiving adjuvant therapy for breast cancer: The IBCSG approach. Annals of Oncology, 8, 825-835. doi: 10.1023/A:1008269715091

Brady, M. J., Cella, D. F., Mo, F., Bonomi, A. E., Tulsky, D. S., Lloyd, S. R.,... Shiomoto, G. (1997). Reliability and validity of the Functional Assessment of Cancer Therapy-Breast Quality of Life instrument. Journal of Clinical Oncology, 15, 974-986.

Brady, M. J., Peterman, A. H., Fitchett, G., Mo, M., \& Cella, D. (1999). A case for including spirituality in quality of life measurement in oncology. Psycho-Oncology, 8, 417-428. doi: 10.1002/(SICI)1099-1611(199909/10)8:5<417::AID-PON398>3.0.CO;2-4

Carver, C. S. (1997). You want to measure coping but your protocol is too long: Consider the Brief COPE. International Journal of Behavioral Medicine, 4, 92-100. doi: 10.1207/s15327558ijbm0401_6

Carver, C. S., Scheier, M. F., \& Weintraub, J. K. (1989). Assessing coping strategies: A theoretically based approach. Journal of Personality and Social Psychology, 56, 267283. doi: 10.1037//0022-3514.56.2.267

Cella, D. F., Tulsky, D. S., Gray, G., Sarafian, B., Linn, E., Bonomi, A., ... Brannon, J. (1993). The Functional Assessment of Cancer Therapy Scale: Development and validation of the general measure. Journal of Clinical Oncology, 11, 570-579.

Curtis, A. J. (2000). Health Psychology. New York: Taylor e Francis Group.

Diener, E., Emmons R. A., Larsen, R. J., \& Griffin, S. (1985). The Satisfaction with Life Scale. Journal of Personality Assessment, 49, 71-75. doi:10.1207/s15327752jpa4901_13

Fabri, A., Carcangiu, M. L., \& Carbone, A. (2008). Histological classification of breast cancer. In E. Bombardieri, G. Bonadonna, \& L. Gianni (Eds.), Breast cancer: Nuclear 
medicine in diagnosis and therapeutic options (pp. 3-14). New York: Springer Berlin Heidelberg.

Faria, J. B., \& Seidl, E. M. F. (2005). Religiosidade e enfrentamento em contextos de saúde e doença: Revisão da literatura. Psicologia: Reflexão e Crítica, 18, 381-389. doi:10.1590/S0102-79722005000300012

Feher, S., \& Maly, R. C. (1999). Coping with breast cancer in later life: The role of religous faith. Psycho-Oncology, 8, 408-416. doi:10.1002/(SICI)10991611(199909/10)8:5<408::AID-PON409>3.0.CO;2-5

*Gall, T. L., Guirguis-Younger, M. G., Charbonneau, C., \& Florack, P. (2009). The trajectory of religious coping across time in response to the diagnosis of breast cancer. PsychoOncology, 18, 1165-1178. doi:10.1002/pon.1495

*Hebert, R., Zdaniuk, B., Schulz, R., \& Scheier, M. (2009). Positive and negative religious coping and well-being in women with breast cancer. Journal of Palliative Medicine, 12, 537-545. doi: 10.1089/jpm.2008.0250

Herrmann, C., Buss, U., \& Snaith, R. P. (1995). HADS-D - Hospital Anxiety and Depression Scale - Deutsche Version [HADS-D - German Version]. Huber, Bern, Switzerland.

Huber S. (2003) Zentralität und Inhalt. Ein neues multidimensionales Messmodell der Religiosität. (Centrality and Contents. A new Multidimensional Measurement Model of Religiosity). Opladen: Leske and Budrich

Koenig, H. G. (2007). Spirituality in patient care: why, how, when, and what. United States of America: Templeton Foundation Press (Original publicado em 2002).

Koenig, H. G. (2008). Medicine, religion and health: Where science and spirituality meet. Pennsylvania: Templeton Foundation Press.

*Laarhoven, H. van, Schilderman, J. Vissers, K. C., Verhagen, C. A. H. H. V. M., \& Prins, J. (2010). Images of God in relation to coping strategies of palliative cancer patients. Journal of Pain and Symptom Management, 40, 495-501. doi:10.1016/j.jpainsymman.2010.02.021

Lazarus, R. S., \& Folkman, S. (1984). Stress, appraisal, and coping. New York: Springer Publishing Company.

Lin, H-R., \& Bauer-Wu, S. M. (2003). Psycho-spiritual well-being in patients with advanced cancer: an integrative review of the literature. Journal of Advanced Nursing, 44, 69-80. doi: 10.1046/j.1365-2648.2003.02768.x

Lovibond, S. H., \& Lovibond, P. F. (1995). Manual for the Depression Anxiety Stress Scales (2nd ed.). Sydney, Australia: Psychology Foundation of Australia.

McNair, D., Lorr, M., \& Droppleman, L. (1992). POMS Manual: Profile of Mood States. San Diego: Edits/Educational and Industrial Testing Service.

Miller, W. R., \& Thoresen, C. E. (2003). Spirituality, religion, and health: An emerging research field. American Psychologist, 58, 24-35. doi: 10.1037/0003-066X.58.1.24

*Morgan, P. D., Gaston-Johansson, F., \& Mock, V. (2006). Spiritual well-being, religious coping, and the quality of life of African American breast cancer treatment: A pilot study. Official Journal of the Association of Black Nursing Faculty in Higher Education, 17, 73-77. 
Muller, M. C., Hoffman, F. S., \& Fleck, P. (2006). A vivência do câncer de mama e a imagem corporal na mulher contemporânea. In B. S. G. Werlang \& M. da S. Oliveira (Orgs.). Temas em Psicologia Clínica (pp.203-208). São Paulo: Casa do Psicólogo.

Muthny, F. A. (1989). Freiburger Fragebogen zur Krankheitsverarbeitung (FKV) (Manual Freiburg Questionnaire of Coping with Illness (FQCI)). Weinheim: Beltz.

Mytko, J. J., \& Knight, S. J. (1999). Body, mind and spirit: towards the integration of religiosity and spirituality in cancer quality of life research. Psycho-Oncology, 8, 439450. doi: 10.1002/(SICI)1099-1611(199909/10)8:5<439::AID-PON421>3.0.CO;2-L

Paiva, G. J. de. (2007). Religião, enfrentamento e cura: Perspectivas psicológicas. Estudos de Psicologia, Campinas, 24, 99-104. doi: 10.1590/S0103-166X2007000100011

Paiva, G. J. de., Zangari, W., Verdade, M. M., Paula, J. R. M. de., Faria, D. G. R. de., Gomes, D. M.,... Gomes, A.(2009). Psicologia da religião no Brasil: A produção em periódicos e livros. Psicologia: Teoria e Pesquisa, 25, 441-446. doi: 10.1590/S010237722009000300019

Panzini, R. G., Rocha, N. S. da, Bandeira, D. R., \& Fleck, M. P. de A. (2007). Qualidade de vida e espiritualidade. Revista de Psiquiatria Clínica, 34, 105-115. doi: 10.1590/S010160832007000700014

Pargament, K. I., Smith, B. W., Koenig, H. G., \& Pereza, L. (1998). Patterns of positive and negative religious coping with major life stressors. Journal for the Scientific Study of Religion, 37, 710 -724. doi: 10.2307/1388152

Pargament, K. I. (1997). The psychology of religion and coping: theory, research, practice. New York: Guilford Press.

Pargament, K. I., Koenig, H. G., \& Perez, L. M. (2000). The many methods of religious coping: Development and initial validation of the RCOPE. Journal of Clinical Psychology, 56, 519-543. doi: 10.1002/(SICI)1097-4679(200004)56:4<519::AIDJCLP6>3.0.CO;2-1

Plante, T. G., \& Boccaccini, M. T. (1997). The Santa Clara Strength of Religious Faith Questionnaire. Pastoral Psychology, 45, 375-387. doi: 10.1007/BF02230993

Ryff, C. D. (1989). Happiness is everything, or is it? Explorations on the meaning of psychological well-being. Journal of Personality and Social Psychology, 57, 10691081. doi: 10.1037//0022-3514.57.6.1069

Schnoll, R. A., Harlow, L. L., \& Brower, L. (2000). Spirituality, demographic and disease factors, and adjustment to cancer. Cancer Practice, 8, 298-304. doi: 10.1046/j.15235394.2000.86006.x

*Schreiber, J. (2011). Image of God: Effect on coping and psychospiritual outcomes in early breast cancer survivors. Oncology Nursing Forum, 38, 293-301. doi:10.1188/11.ONF.293-301

Shaw, C. (1999). A framework for the study of coping, illness behavior and outcomes. Journal of Advanced Nursing, 29, 1246-1255. doi: 10.1188/11.ONF.293-301

Silva, L. C. da. (2008). Câncer de mama e sofrimento psicológico: Aspectos relacionados ao feminino.Psicologia em Estudo, 13, 231-237. doi: 10.1590/S1413-73722008000200005

Snyder, C. R., \& Dinoff, B. L. (1999). Coping: Where have you been? In C. R. Snyder (Ed.), The psychology of what works (pp.3-19). New York: Oxford University Press. 
Soothill, K., Morris, S. M., Harman, J. C., Thomas, C., Francis, B., \& Mclllmurray, M. B. (2000). Cancer and faith. Having faith - does it make a difference among patients and their informal carers? Scandinavian Journal of Caring Sciences, 16, 256-263. doi: 10.1046/j.1471-6712.2002.00097.x

Sousa, P. L. R., Tillman, I. A., Horta, C. L., \& Oliveira, F. M. de (2004). A religiosidade e suas interfaces com a medicina, a psicologia e a educação: O estado da arte. In E. F. B. Teixeira, M. C. Muller, \& J. D. T. da Silva (Eds.), Espiritualidade e qualidade de vida (pp.51-64). Porto Alegre: EDIPUCRS.

Speechley, V., \& Rosenfield, M. (2000). Tudo sobre câncer. São Paulo: Andrei.

Stefanek, M., McDonald, P. G., \& Hess, S. A. (2005). Religion, spirituality and cancer: Current status and methodological challenges. Psycho-Oncology, 14, 450-463. doi: $10.1002 /$ pon. 861

Straub, R. O. (2005). Psicologia da saúde (R. C. Costa, Trad.). Porto Alegre: Artmed.

*Thuné-Boyle, I. C. V., Stygall, J. A., Keshtgar, M. R., \& Newman, S. P. (2006). Do religious/spiritual coping strategies affect illness adjustment in patients with cancer? A systematic review of the literature. Social Science \& Medicine, 63,151-164. doi: 10.1016/j.socscimed.2005.11.055

Thuné-Boyle, I. C. V., Stygall, J. A., Keshtgar, M. R., Davidson, T. I., \& Newman, S. P. (2011). Religious coping strategies in patients diagnosed with breast cancer in the UK. Psycho-Oncology, 20, 771-782. doi: 10.1002/pon.1784

Van der Vem, J. A. (1998). God reinvented? A theological search in texts and tables. Leiden, The Netherlands: Brill.

Vickberg, S. M. (2003). The Concerns About Recurrence Scale (CARS): A systematic measure of women's fears about the possibility of breast cancer recurrence. Annals of Behavioral Medicine, 25,16-24. doi: 10.1207/S15324796ABM2501_03

Wanderley, K. da S. (2003). Aspectos psicológicos do câncer de mama. In M. M. M. J. de Carvalho (Ed.), Introdução à Psiconcologia (pp.95-101). São Paulo: Editora Livro Pleno.

Ware, J. E. Jr., \& Sherbourne, C. D. (1992). The MOS 36-item short-form health survey (SF36). I. Conceptual framework and item selection. Medical Care, 30, 473-483. doi: 10.1097/00005650-199206000-00002

Yanez, B., Edmondson, D., Stanton, A. L., Park, C. L., Kwan, L., Ganz, P. A., \& Blank, T.O. (2009). Facets of Spirituality as Predictors of Adjustment to Cancer: Relative Contributions of Having Faith and Finding Meaning. Journal of Consulting and Clinical Psychology, 77, 730-741. doi: 10.1037/a0015820

*Zwingmann, C., Wirtz, M., Müller, C., Körber J., \& Murken, S. (2006). Positive and negative religious coping in German breast cancer patients. Journal of Behavioral Medicin, 29, 533-547. doi: 10.1007/s10865-006-9074-3

*Zwingmann, C., Müller, C., Körber, J., \& Murken, S. (2008). Religious commitment, religious coping and anxiety: a study in German patients with breast cancer. European Journal of Cancer Care, 17, 361-370. doi:10.1111/j.1365-2354.2007.00867.x 